\title{
Fetal Complications in COVID-19 Infected Pregnant Woman: A Systematic Review and Meta-Analysis
}

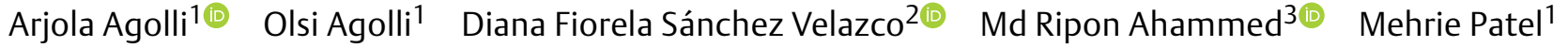 \\ Jose Cardona-Guzman ${ }^{4}$ Radhika Garimella ${ }^{5}$ Ratcha Rummaneethorn ${ }^{6}$ Seema Bista ${ }^{1}$ \\ Rafael Abreu ${ }^{4}$ Nikole Czapp ${ }^{4}$ Manuel Garcia ${ }^{4}$ \\ ${ }^{1}$ Division of Clinical \& Translational Research, Larkin Health System, \\ Address for correspondence Arjola Agolli, MD, MBA, Division of \\ South Miami, Florida, United States \\ ${ }^{2}$ Cayetano Heredia Peruvian University, San Martín de Porres, Peru \\ ${ }^{3}$ National Institute of Cardiovascular Diseases, Dhaka, Bangladesh \\ ${ }^{4}$ Larkin Community Hospital System, South Miami, Florida, United \\ Clinical \& Translational Research, Larkin Health System, 7000 SW 62nd \\ Ave, Suite 601, South Miami, FL 33143, United States \\ (e-mail: arjolamusta@hotmail.com). \\ States \\ ${ }^{5}$ Dr. N.T.R University of Health Sciences, India \\ 6 Metropolitan Hospital, New York, United States \\ Avicenna J Med 2021;11:200-209.
}

\begin{abstract}
Background Pregnancy is an immunocompromised state and, for this reason, a pregnant woman is at a higher risk of getting infected as compared with a healthy individual. There is limited data available regarding the impact of COVD-19 on pregnancy; however, the case of miscarriage due to placental infection caused by severe acute respiratory syndrome coronavirus 2 (SARS-CoV-2) in second trimester has already been reported.

Methods We searched for all published articles in PubMed, Science Direct, Cochrane, Scopus, and Embase. The literature search produced 167 relevant publications; 67 manuscripts were further excluded because they did not satisfy our inclusion criteria. Out of the remaining 100 articles, 78 were excluded after full text screening. Therefore, a total of 22 articles were eligible for review in our study.

Results Overall, these 22 studies included a total of 7,034 participants: 2,689 (38.23\%) SARS-CoV-2 positive pregnant women, of which 2,578 (95.87\%) were laboratory confirmed and 111 (4.13\%) were clinically diagnosed. Among the positive

Keywords

- coronavirus

- COVID-19

- pregnancy loss

- abortion

- pregnancy patients, there were $174(6.47 \%)$ cases of abortion, of them 168 (96.55\%) were spontaneous abortions and $6(3.45 \%)$ were missed. Most patients either reported mild symptoms of fever, cough, fatigue, and anosmia or they presented asymptomatic.

Conclusion Additional investigation and rigorous research are warranted to confirm placental pathology mechanisms concerning COVID-19 to protect maternal and fetal health.
\end{abstract}

published online

November 15, 2021
DOI https://doi.org/ $10.1055 / \mathrm{s}-0041-1736540$ ISSN 2231-0770.

\footnotetext{
C 2021. Syrian American Medical Society. All rights reserved. This is an open access article published by Thieme under the terms of the Creative Commons Attribution-NonDerivative-NonCommercial-License, permitting copying and reproduction so long as the original work is given appropriate credit. Contents may not be used for commercial purposes, or adapted, remixed, transformed or built upon. (https://creativecommons.org/ licenses/by-nc-nd/4.0/)

Thieme Medical and Scientific Publishers Pvt. Ltd., A-12, 2nd Floor, Sector 2, Noida-201301 UP, India
} 


\section{Introduction}

COVID-19 infection is spread by respiratory droplets and is highly contagious. ${ }^{1}$ It was first reported in Wuhan, China in December 2019 and the COVID-19 pandemic was declared by the World Health Organization on March 11, 2020. ${ }^{2}$ COVID-19 enters the body via the nasal passage and infects pulmonary cells via the severe acute respiratory syndrome coronavirus 2 (SARS-CoV-2) receptor angiotensin-converting enzyme 2 (ACE2) and uses transmembrane serine protease 2 (TMPRSS2) for $S$ protein priming. ${ }^{3}$ Infection with SARS$\mathrm{CoV}-2$ is followed by viral replication and release of the virus, causing pyroptosis (inflammation-mediated programmed cell death occurring in response to a pathological stimulus). ${ }^{4}$ Coronavirus is enveloped, nonsegmented, positive-sense ribonucleic acid usually causing respiratory distress in the infected patient. ${ }^{5}$ However, evidence shows that coronavirus can cause harmful clinical effects on a wide spectrum of bodily systems. Since pregnancy is an immunocompromised state, a pregnant woman is at a higher risk of getting infected as compared with a healthy individual. ${ }^{1}$ The modulations of the maternal immune system in pregnancy may affect the response to infections, and specifically to viruses. ${ }^{6}$

Mechanisms of damages of COVID-19 during pregnancy could be: a decrease in circulating natural killer (NK) cells ${ }^{7}$; increased progesterone hormone as a steroid hormone that has immunomodulatory properties ${ }^{8}$; alterations in the innate immune system, including the pattern recognition receptors Toll-like receptors (TLRs) ${ }^{9}$; a shift in CD4+ T cell population toward the Th2 phenotype over Th $1^{10}$; also pregnancy is a hypercoagulable state with increased thrombin production and an increase in intravascular inflammation ${ }^{11}$; and the current reports indicate clinical manifestations of both widespread microvascular as well as large vessel thrombosis in patients infected with COVID-19. ${ }^{12}$ But the fetal damages are explained by SARS-CoV-2 found on reverse transcriptionpolymerase chain reaction of swabs and biopsies following a spontaneous fetal loss at 19 -week gestation ${ }^{13}$ and in placental and umbilical cord biopsies. ${ }^{14}$ Mechanisms of viral invasion of the placenta have yet to be established.

Outcomes in pregnant women with SARS-CoV-2 infection appear less severe compared with SARS and Middle East respiratory syndrome. ${ }^{15}$ American Centers for Disease Control states that although hospitalization in the intensive care unit of COVID-19 affected pregnant patient is higher than nonpregnant, the mortality rate is similar to other nonpregnant patients. Since there is evidence that COVID-19 affects blood coagulation factors, it has the potential of adverse effect on pregnancy, particularly with the inherent hypercoagulability of gestation. ${ }^{2}$ There had been a systematic review of published reports on COVID-19 which reported higher rates of preterm birth, preeclampsia, and perinatal death. The lack of data on spontaneous abortion due to COVID-19 in the first trimester prevents the inference of conclusive evidence for the effects of this infection during early pregnancy. But due to the scarcity of reliable data and misreporting of the information by the media has led pregnant women to take drastic choices such as voluntary abortion. ${ }^{5}$ The wide range of COVID-19 symptoms, high rate of asymptomatic forms, and poor accuracy of nasopharyngeal swab testing have been the main barriers for understanding the prevalence of its infection and impact on a pregnant woman and the fetus. ${ }^{5}$ Though we are aware that many viral infections are harmful to the fetus during the first trimester of pregnancy, what is the impact of COVID-19 on the fetus of the first trimester is still unknown. ${ }^{5}$ There was a lack of study and reliable data to find the effect of COVID-19 on the first trimester and second trimester; however, there were some case reports of newborns with fetal distress requiring intensive care unit and stillbirth of COVID-19 affected pregnant women in the third trimester suggesting the possibility of pathology related to the placenta. ${ }^{16}$ According to Yan et al, a retrospective study where 116 pregnant women were included resulted that SARS-CoV-2 infections during pregnancy is not associated with an increased risk of spontaneous abortion and spontaneous preterm birth. There is no evidence of vertical transmission of SARS-CoV-2 infection when the infection manifests during the third trimester of pregnancy. ${ }^{17}$ The same conclusion was reported by the Cosma et al case-control study where 225 women were studied. COVID-19 did not seem to predispose to early pregnancy loss; its cumulative incidence did not show a difference between women with spontaneous abortion and women with ongoing pregnancy. COVID-19 appears to have a favorable maternal course at the beginning of pregnancy, consistent with what has been observed during the second and third trimesters. ${ }^{5}$ In the Baud et al case report, the miscarriage during the first trimester due to SARS-CoV-2 infection appears related to placental infection as no other cause of miscarriage was identified. Limited data are available regarding the impact of COVD-19 on pregnancy; however, the case of miscarriage due to placental infection caused by SARS-CoV-2 in the second trimester has already been reported more than once. ${ }^{16,18}$ An increasing number of miscarriage cases were reported during this pandemic. These cases of missed miscarriages were diagnosed clinically, supported by ultrasonographic evidence, before being diagnosed with COVID-19 infection. This could happen even in those cases where the patients were relatively asymptomatic or manifested mild symptoms of COVID-19 infection. However, the cause of the miscarriage was unknown, and SARSCoV-2 infection as the causal factor could not be completely ruled out. ${ }^{19}$ The position for pregnant women on this spectrum is unclear. The immune system adapts during pregnancy to allow for the growth of a semiallogenic fetus. ${ }^{20}$

Since December 2019 (when the pandemic started), several case studies and cohort studies have described the presentation and clinical course of COVID-19 in pregnancy. Therefore, the main aim of this study is to understand the role of COVID-19 infection in causing pregnancy complications specifically fatal outcomes.

\section{Methods}

This literature review is done for the period January 1, 2020 up to July 2021. We searched for articles in PubMed, Science 
Direct, Cochrane, Scopus, and Embase. We used the following search terms "Pregnancy complications and/or COVID-19" and/or Fetal complications and/or SARS-CoV-2 and/or Pregnancy Complications and/or SARS-CoV-2. The search terms were kept broad to encompass all possibilities for applicable studies. Only articles published in the English language were included. Duplicates were removed manually. After eliminating duplicates, three investigators (D.S., R.A., and O.A.) independently reviewed all titles and abstracts. The full texts of articles regarded as potentially eligible for consideration were extracted and screened for further analysis. Thereafter, eligible articles were selected for final analysis according to predefined inclusion and exclusion criteria. Disagreements between the authors were resolved through consensus and active discussion. The exclusion criteria consisted of review articles, animal studies, elective abortion, studies published in a language other than English, and the absence or unclear reporting of pregnancy complication status in COVID-19 pregnant patients.

\section{Results}

The literature search produced 90 relevant publications in the PubMed database, 5 on ScienceDirect, and 72 on Scopus, and no results of Embase or Cochrane. All Google Scholar's results were repeated. After reviewing 167 articles manually by abstract screening, 67 manuscripts were further excluded because they did not satisfy our inclusion criteria. Out of the remaining 100 articles, 78 were excluded after full text screening. Therefore, 22 articles were eligible for review in our study. Details of included studies are listed in the table. The majority of papers arose from Asia. Of all of the studies included, 4 were case series reports, 6 case reports, 7 cohort studies, 4 cross-sectional, and 1 case-control study. Please find the information on all fatal outcome pregnancies reported in the literature that we included in our final quantitative analysis in -Table 1.

Overall, these 22 studies included a total of 7,034 participants: 2,689 (38.23\%) SARS-CoV-2 positive pregnant women, of which 2,578 (95.87\%) were laboratory confirmed and $111(4.13 \%)$ were clinically diagnosed. The clinical symptoms among pregnant women with laboratory-confirmed SARS$\mathrm{CoV}-2$ infection are presented in the table.

\section{Symptoms}

Symptoms most commonly reported were four: fever, cough, fatigue, and anosmia. Out of 3,512 patients, $15.19 \%$ had fever. $^{5,16-18,22-24,26-28,30,31}$ Out of 2,155, 28.19\% presented with a cough and $16.6 \%$ developed fatigue from 307 cases. ${ }^{5,16,17,19,22-24,26-28,30,31}$ Also, from 2,360 cases, $4.32 \%$ reported anosmia. ${ }^{5,16,18,24,26,27,30}$ Six studies reported asymptomatic patients with COVID-19, so $34.3 \%$ from 1,892 cases. $^{19,23,24,26,28}$ There were three studies in which 89.11\% reported mild disease of COVID-19. ${ }^{24,26,28}$

Only one study compared clinical symptoms between COVID-19 positive early pregnancy loss and pregnant group during the first trimester: fever, anosmia, ageusia, cough, arthralgia, diarrhea, and no cases of pneumonia were reported. Also, was noted no difference in the incidence of symptoms between the two groups. ${ }^{5}$

\section{Vertical Transmission of SARS-COV-2 and Spontaneous Abortion}

Martinez-Perez et al observed a significant increase in the stillbirth rate in the univariate analysis. It is an important point to highlight that two case reports confirmed positive SARS-CoV-2 in placental tissue samples and amniotic fluid. ${ }^{34,37}$ Among the positive patients, there were 174 (6.47\%) cases of abortion, of them 168 (96.55\%) were spontaneous abortions and 6 (3.45\%) were missed abortions. Comparing to United States 2021 abortion rate, which is reported as $20.8 \%{ }^{38}$ there were also four cases of induced abortion owing to patient's concerns about COVID-19 and two cases of ectopic pregnancy. We have excluded these 6 cases from the analysis. Otherwise, three studies reported the use of lopinavir-ritonavir as therapy, so $6.23 \%$ of 593 cases. $^{21,28,31}$

\section{Assessment of Study Quality}

For the quality assessment of included studies the Newcastle-Ottawa Scale was employed to ascertain the quality of studies by two reviewers (D.S.) and (R.A.). They independently assessed the methodologic quality of each study included: 7 cohort studies, 4 cross-sectional, and 10 observational studies were evaluated for the following three domains: quality of selection of cohorts (4 stars), comparability of cohorts (2 stars), and assessment of outcome (3 stars). One case-control study was assessed for quality of selection of cases and controls (4 stars), comparability of cases and controls (2 stars), and ascertainment of exposure (3 stars). We considered a total of 7 out of 9 stars to be a low risk of bias, 4 to 6 stars to be a moderate risk, and less than 4 stars to be a high risk of bias. In case of a disagreement, reviewers reached consensus by discussion with a third reviewer. The risk of bias assessment is shown in - Table 2.

\section{Discussion}

SARS-CoV-2 Complicates the Pregnancy Utilizing ACE2 Receptor and Consequent Activation of the TMPRSS2 Enzyme

Coronavirus disease's clinical spectrum can range from asymptomatic presentation to pneumonia, heart failure, kidney injury, and eventually death. The SARS-CoV-2 utilizes the ACE2 receptor and consequent activation of the TMPRSS2 enzyme to gain entry into host cells and trigger an exaggerated inflammatory response via the release of cytokines ("cytokine storm"). These results in a severe form of COVID-19 infection which is associated with increased mortality and morbidity. An intact immune system is responsible for clearing infected cells and preventing further replication of the virus. A weakened immune system in pregnancy impacts viral clearance and therefore increases the susceptibility to develop an infection with SARS-CoV-2. Factors such as a preference of the humoral response over the cellmediated response, a decrease in circulating NK cells, and alterations in the immune system and pattern recognition 


\begin{tabular}{|c|c|c|c|c|c|c|c|c|c|c|c|}
\hline 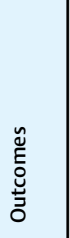 & 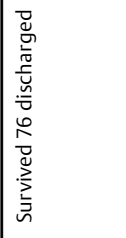 & 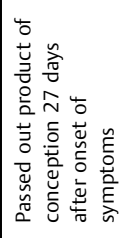 & 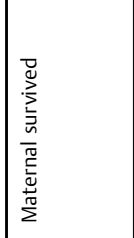 & 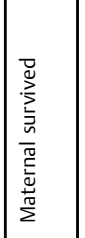 & 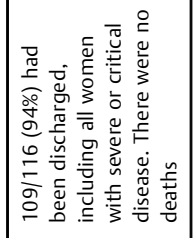 & 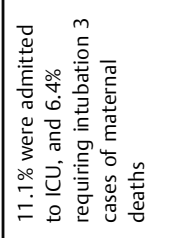 & 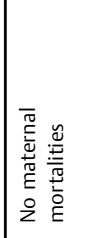 & 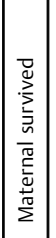 & 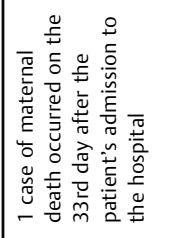 & 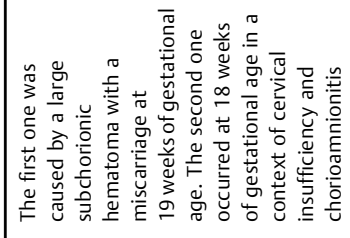 & 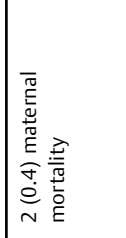 \\
\hline 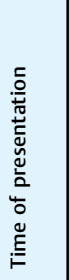 & 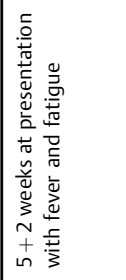 & 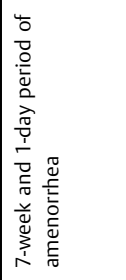 & 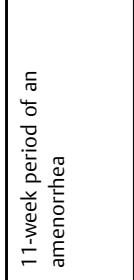 & 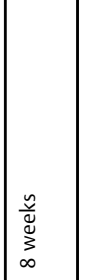 & 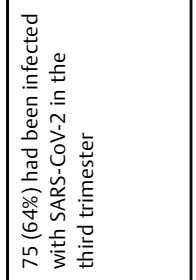 & 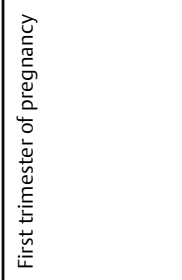 & 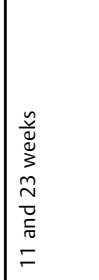 & $\mid$ & 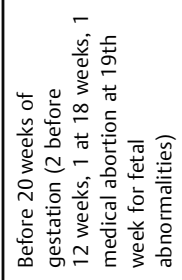 & 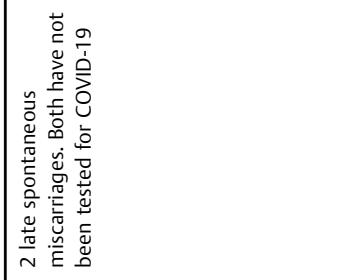 & 文 \\
\hline 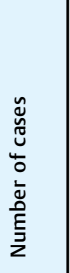 & 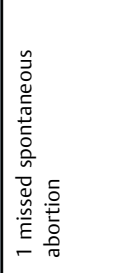 & 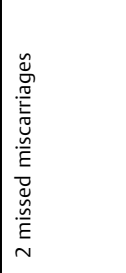 & & 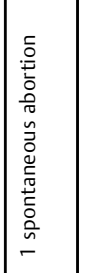 & 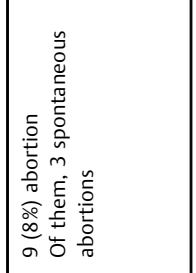 & 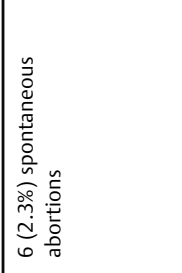 & 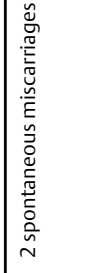 & 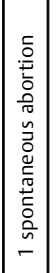 & 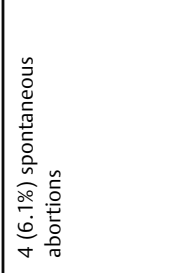 & 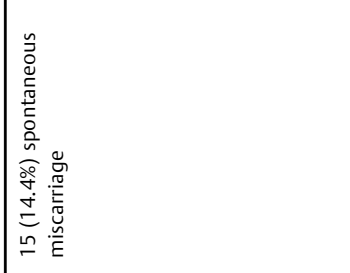 & 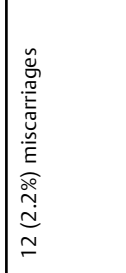 \\
\hline 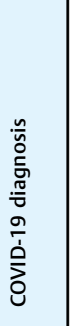 & 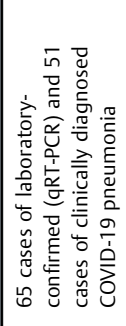 & 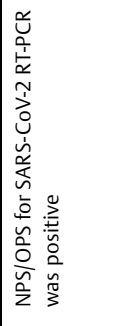 & 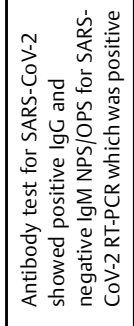 & 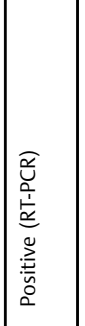 & 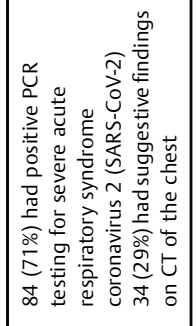 & 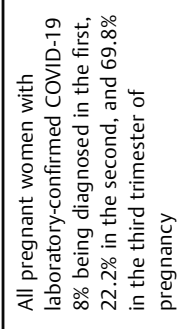 & 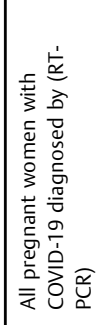 & 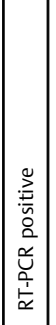 & 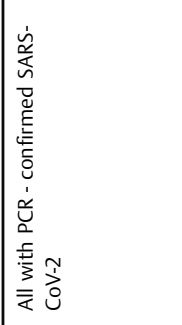 & 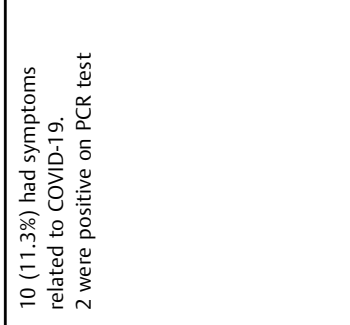 & 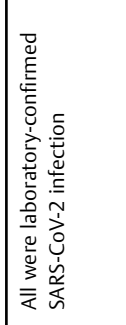 \\
\hline 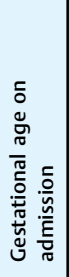 & 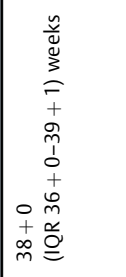 & 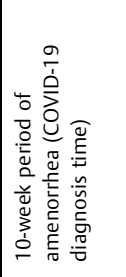 & 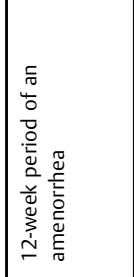 & 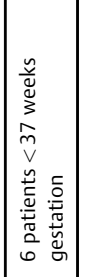 & 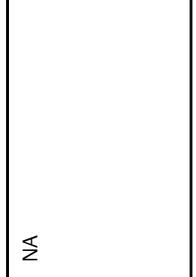 & 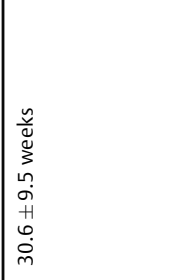 & 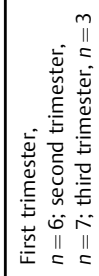 & 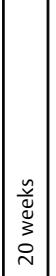 & 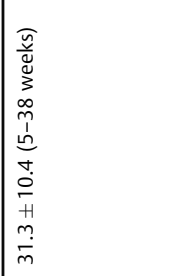 & 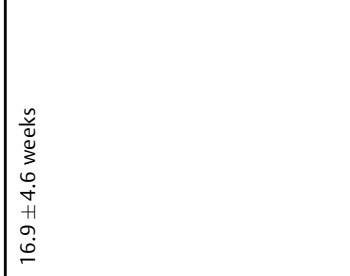 & 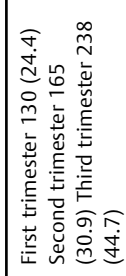 \\
\hline 芩 & 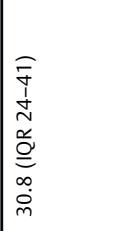 & 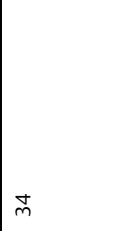 & $\stackrel{\infty}{m}$ & $\frac{\pi}{z}$ & 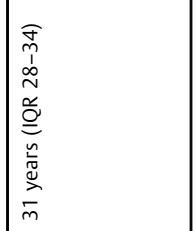 & 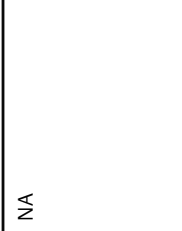 & $\mid \begin{array}{l}0 \\
\stackrel{\sim}{\sim} \\
\tilde{N}\end{array}$ & 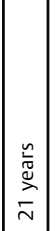 & $\mid \begin{array}{l}\stackrel{\sim}{N} \\
0 \\
0 \\
m \\
m \\
\dot{m} \\
\dot{m}\end{array}$ & \begin{tabular}{|l}
$-\dot{j}$ \\
+ \\
0 \\
$\ddot{m}$ \\
$\dot{m}$
\end{tabular} & 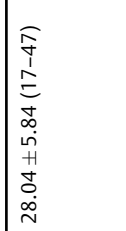 \\
\hline 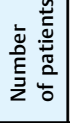 & 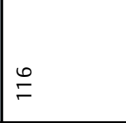 & N & & $\wedge$ & $\stackrel{\infty}{\rightleftharpoons}$ & $\underset{\infty}{\infty}$ & $\stackrel{\circ}{\circ}$ & - & 8 & $\stackrel{ \pm}{ \pm}$ & 䓟 \\
\hline 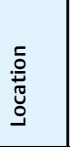 & 莺 & 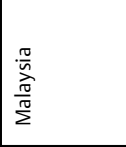 & & 颃 & 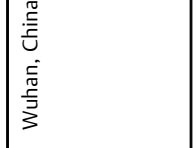 & 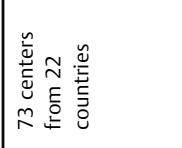 & 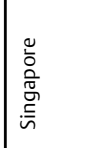 & 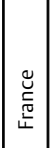 & 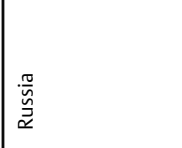 & 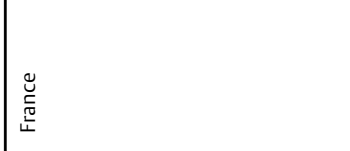 & $\mid \begin{array}{l}\overrightarrow{\vec{Q}} \\
\text { 咅 }\end{array}$ \\
\hline 蒿 & 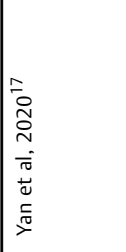 & 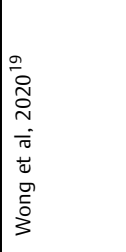 & & 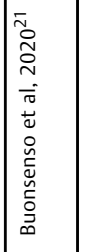 & 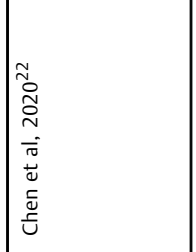 & 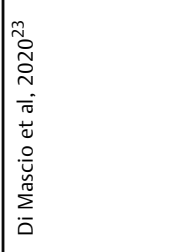 & 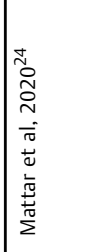 & 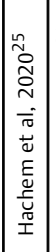 & 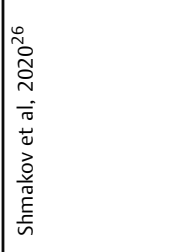 & 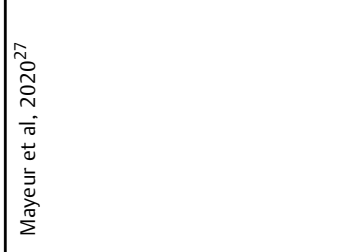 & 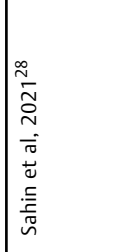 \\
\hline
\end{tabular}




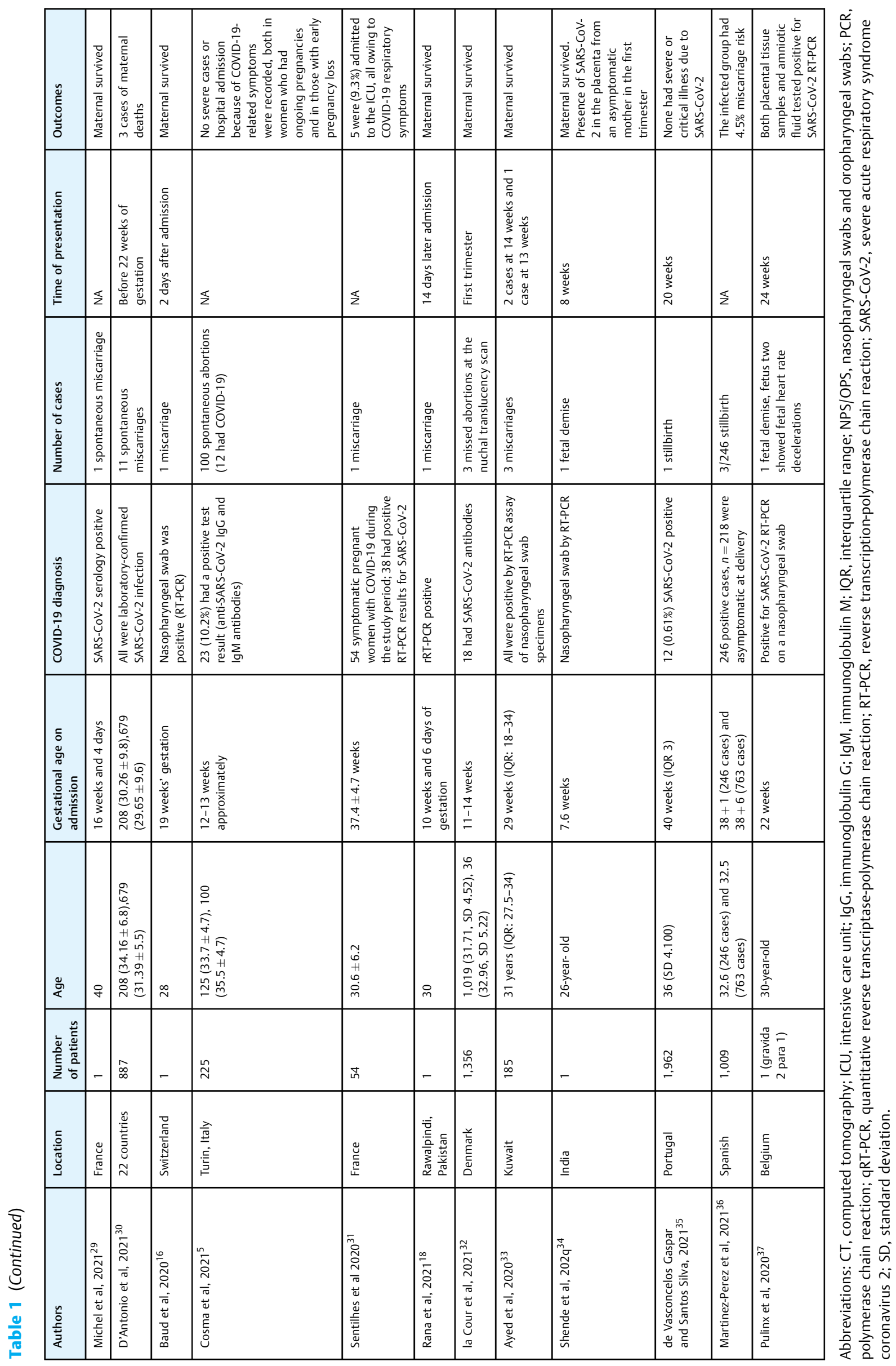




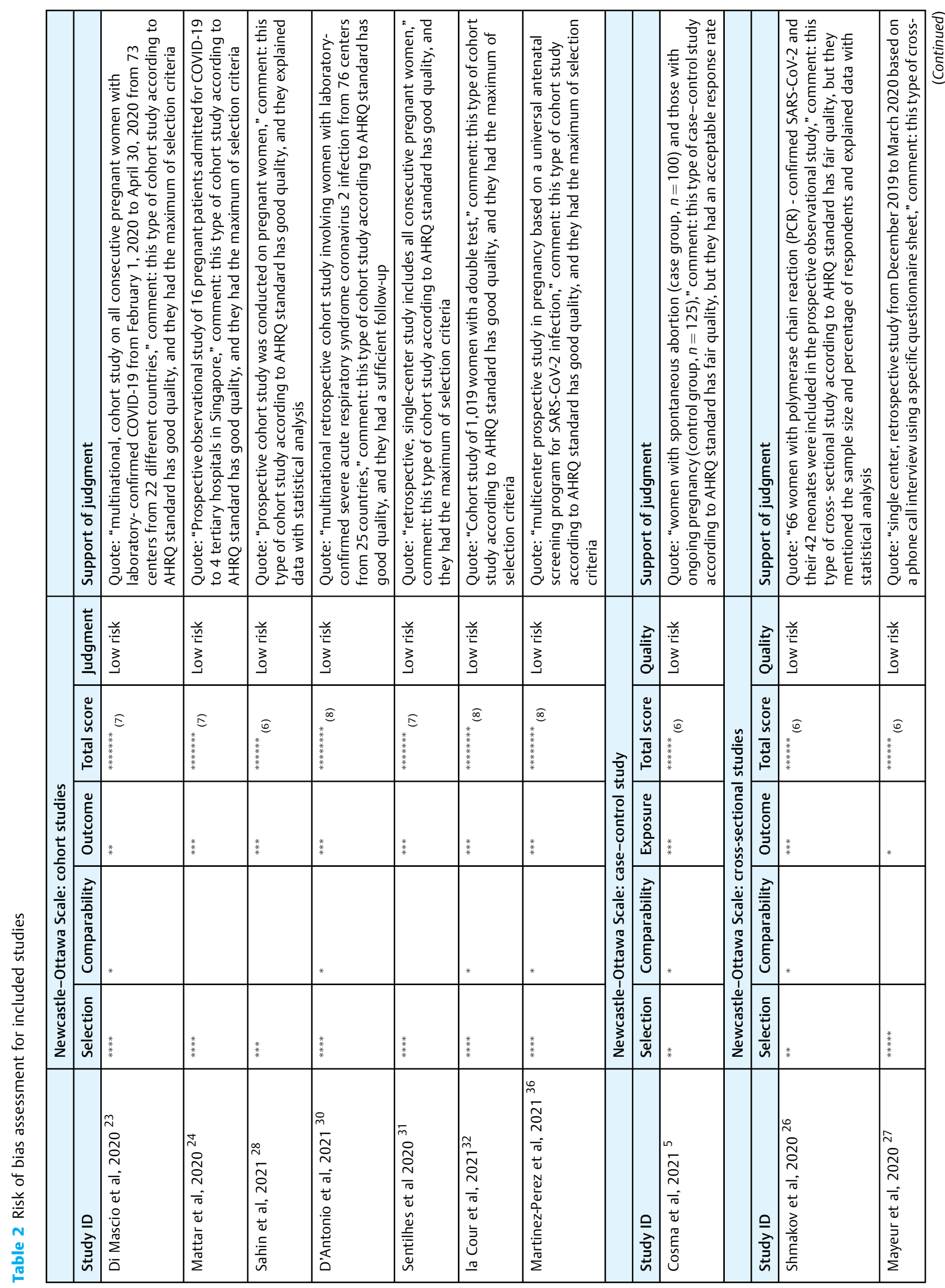




\begin{tabular}{|c|c|c|c|c|c|c|c|c|c|c|c|c|c|c|}
\hline 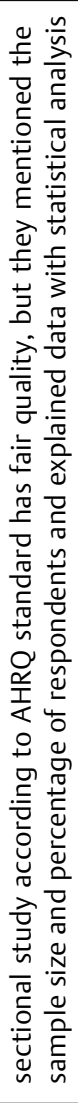 & 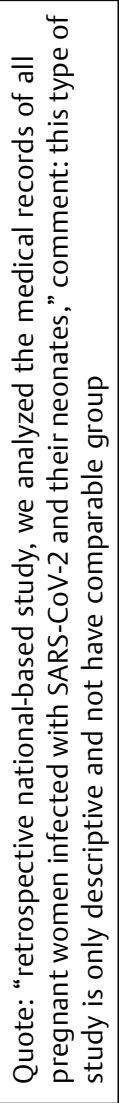 & 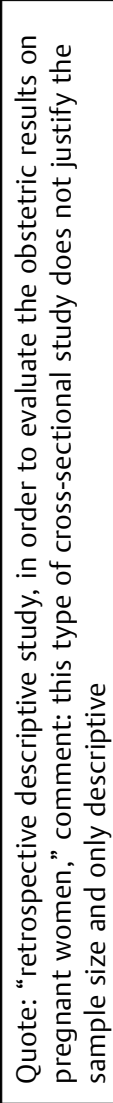 & & 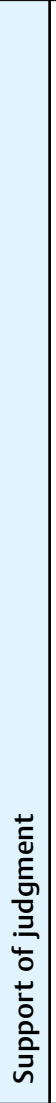 & 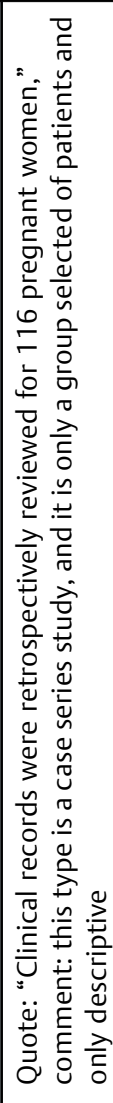 & 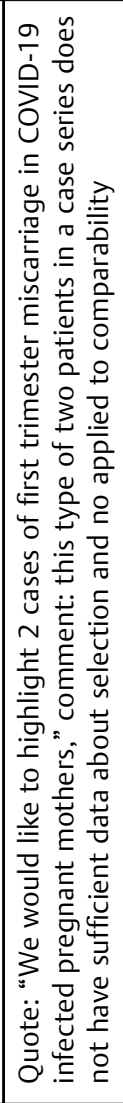 & 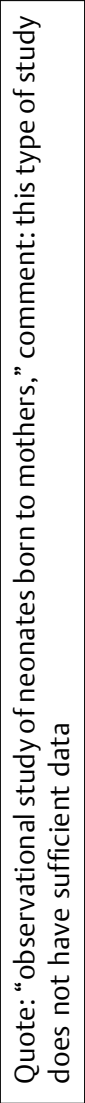 & 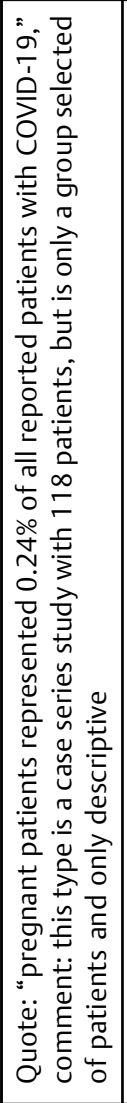 & 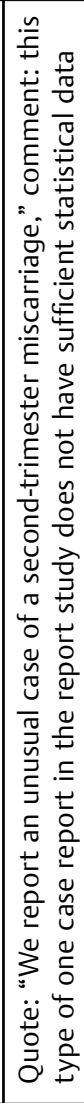 & 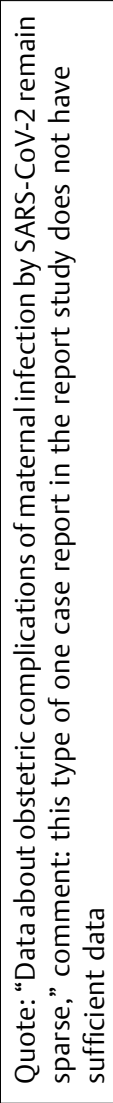 & 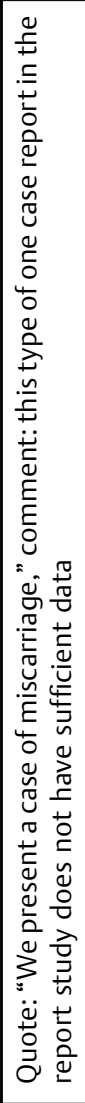 & 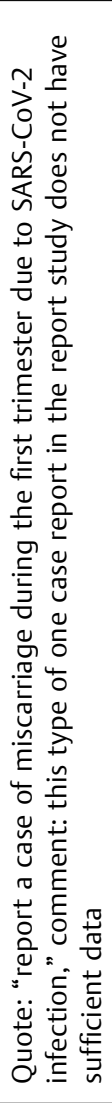 & 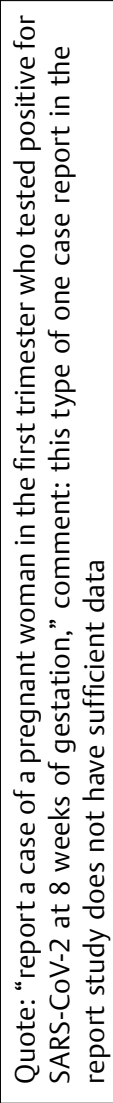 & 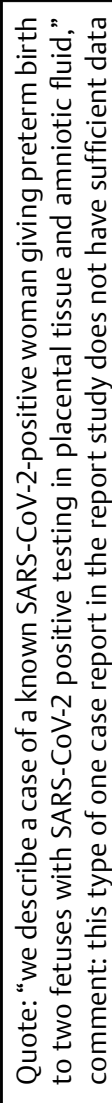 \\
\hline & 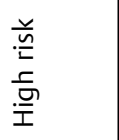 & $\begin{array}{l}\frac{\breve{m}}{\underline{\underline{L}}} \\
\underline{\underline{\underline{I}}}\end{array}$ & & $\begin{array}{l}\frac{\vec{\Xi}}{\pi} \\
\frac{0}{0}\end{array}$ & 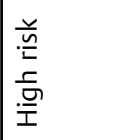 & 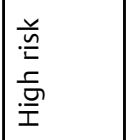 & 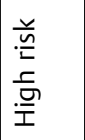 & $\begin{array}{l}\text { 旁 } \\
\text { 咅 }\end{array}$ & 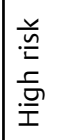 & 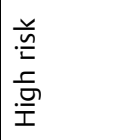 & $\begin{array}{l}\text { 旁 } \\
\text { 咅 }\end{array}$ & $\begin{array}{l}\text { 产 } \\
\text { 馬 } \\
\underline{\underline{\underline{I}}}\end{array}$ & 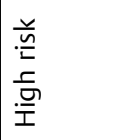 & 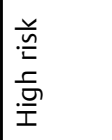 \\
\hline & 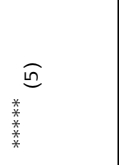 & $\underset{\substack{\text { m } \\
* \\
*}}{ }$ & $\begin{array}{l}\frac{\tilde{y}}{\bar{n}} \\
\bar{z} \\
\frac{\tilde{n}}{\tilde{T}} \\
\frac{0}{0}\end{array}$ & $\begin{array}{l}\frac{0}{\vdots} \\
\stackrel{u}{n} \\
\frac{\pi}{0} \\
\circ\end{array}$ & 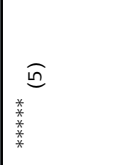 & $\underset{*}{\mathbb{a}}$ & ${ }_{*} \bar{\Xi}$ & $\widehat{\mathbb{a}}$ & 0 & 0 & 0 & 0 & 0 & 0 \\
\hline & 蕃 & 絭 & $\begin{array}{l}\bar{\omega} \\
\hat{0} \\
\text { ¿ }\end{array}$ & $\begin{array}{l}\overline{\bar{u}} \\
0 \\
\dot{\hat{x}} \\
\text {. }\end{array}$ & $\begin{array}{l}* \\
* \\
*\end{array}$ & * & * & * & & & & & & \\
\hline & & & 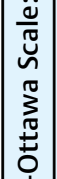 & 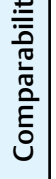 & & & & & & & & & & \\
\hline & * & & 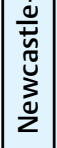 & 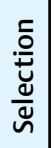 & * & * & & * & & & & & & \\
\hline & 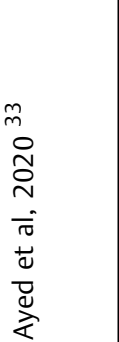 & 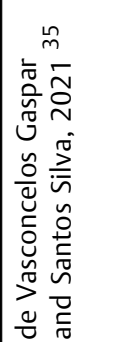 & & 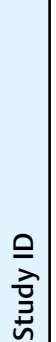 & 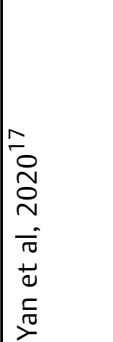 & 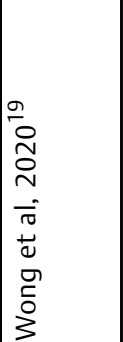 & 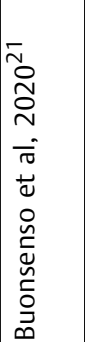 & 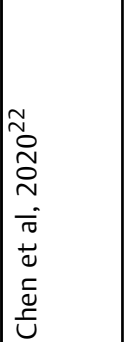 & 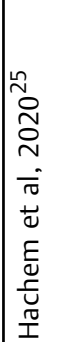 & 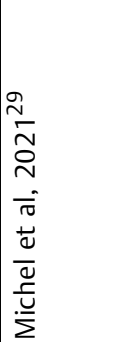 & 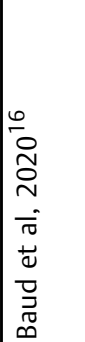 & 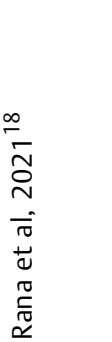 & 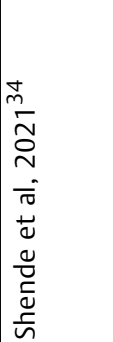 & 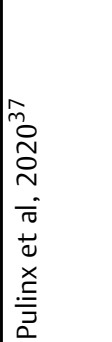 \\
\hline
\end{tabular}


receptors such as the TLRs result in a decreased response to coronavirus disease..$^{7-10,39}$ During pregnancy, the presence of a fetus alters respiratory function as there is an increase in tidal volume, decreased functional residual capacity due to a reduction in chest volume, and a reduction in the total lung capacity. A reduced total lung capacity impairs the lung's ability to clear infections, therefore increasing the susceptibility to develop severe respiratory secretions. $^{39}$

\section{Miscarriage Likely Occurred due to Placental Abruption and Maternal Preeclampsia with Thrombocytopenia and Coagulopathy}

Pregnant women demonstrated enhanced levels of the ACE 2 receptors on organs, such as the placenta, uterus, and kidneys, to allow for proper fetal growth and regulation of angiotensin-II levels. This high expression of ACE2 reveals that the SARS-CoV-2 virus can potentially infect the placenta and result in placental dysfunction and severe pregnancy complications. ${ }^{40}$ Wong et al discuss two cases of first trimester miscarriages in pregnant mothers infected with COVID-19. Although SARS-CoV-2 follows vertical transmission and interacts with fetal ACE2 receptors resulting in fetal death and abortion, the two patients in the study by Wong et al reported no signs of vertical transmission. Reports indicate that the spontaneous miscarriage in both these patients resulted from SARS-related hypoxic respiratory illness. ${ }^{19}$ In addition, Wastnedge et al discuss a series of case reports studying the placentas of pregnant women infected with COVID-19 sustaining miscarriage in the second trimester. A high number of SARS-CoV-2 receptors were expressed in the placental and umbilical cord biopsies in these patients. Reports indicate that the miscarriage likely occurred due to placental abruption and maternal preeclampsia with thrombocytopenia and coagulopathy. Also, electron microscopy in these patients revealed particles of the virus in the cytosol of placental cells. $^{39}$ In addition, Poisson and Pierone's case report indicates extensive fetal vascular malperfusion and parenchymal infarcts resulting in a severe loss of a significant percentage of chorionic villi in the placental examination. ${ }^{41}$

Chowdhury et al's study evaluate 12 cases of miscarriage, diagnosed by an ultrasound scan, at 11 weeks or more due to COVID-19 from March 2020 to July 2020 in a single hospital in Dhaka, Bangladesh. The miscarriage was supposed to be due to SARS-CoV-2 virus-induced damage of the placental barrier via hypoxemia. The miscarriage resulted from viral damage and the consequent induction of a placental inflammatory reaction, acute chorioamnionitis, and intervillous. ${ }^{1}$ Yet, the effect sizes of all of these studies are too small to statistically conclude that the SARSCoV-2 virus is the main culprit in causing miscarriages in pregnant women.

As discussed earlier, SARS-CoV-2 utilizes the ACE2 and the resultant activation of the TMPRSS2 enzyme to gain entry into host cells leading to the coronavirus disease.

\section{COVID-19 Infection in Pregnant Women May More Likely Cause Late Pregnancy Complications and Vertical Transmission}

However, overall, evidence reported so far regarding COVID19 infection and pregnancy demonstrates that the TMPRSS2 enzyme may only be expressed after 24 weeks of pregnancy and only in the extravillous trophoblast. As a result, COVID19 infections in pregnant women may more likely cause late pregnancy complications and vertical transmission as opposed to problems in the first trimester, such as spontaneous or missed abortions. Furthermore, the mild increase in the spontaneous or missed abortions rate may not be because of the SARS-CoV-2 virus itself but due to individual's intense physical and mental stresses from the pandemic. These stressors then cause a release of large amounts of cortisol, which could potentially affect the pregnancy, especially in the early trimester. Yet, at this time, there is limited data available regarding the vertical transmission of SARS-CoV-2 infection. Although recent studies have reported cases of anti-SARS-CoV-19 immunoglobulins in newborns, there are limitations, such as the lack of placental and amniotic fluid examination. $^{42}$

Pregnancy is a vulnerable period, particularly the early period; therefore, it is essential to be vigilant and provide the best maternal care to ensure successful maternal and fetal outcomes. However, as the COVID-19 pandemic is still in progress worldwide, it is vital to assess more extensive studies from a wide range of patient populations and health care settings.

\section{Lessons Learned}

One of the main crucial lessons learned from conducting this comprehensive literature review is that the early pregnancy complications, such as spontaneous or missed abortions, may not have been increased by the COVID-19 infection. As demonstrated by a few studies, the main reason for an increased rate of spontaneous or missed abortions, if any, is due to the environment of the pandemic itself, affecting health care quality and access. ${ }^{43}$ As a result, this increased rate was mainly observed in minority populations, such as African American populations, where there already exists a severe health care disparity. ${ }^{43}$ Thus, to effectively lower the rate of fatal fetal complications in COVID-19-infected pregnant women and maternal mortality, it is best to address the public health aspect of the COVID-19 pandemic instead of clinical management of COVID-19 itself.

\section{Strengths}

We conducted a sensitive and comprehensive search strategy to reduce the risk of missing relevant studies. We adhered to rigorous quality appraisal, which was independently assessed by pairs of reviewers and discrepancies solved by consensus.

\section{Limitations}

Our literature search was restricted to publications in English. Although we included a comprehensive number of 
outcomes, we cannot rule out the possibility that some associations were spurious.

\section{Conclusion}

In conclusion, many current studies included in our comprehensive literature review have shown that there is no increased risk of fetal fatal outcomes in pregnant women infected with COVID-19 infection. Pregnant patients can present with mild disease symptoms such as fever, cough, fatigue, and anosmia. However, almost half of the infected pregnant women were asymptomatic. The expressions of the TMPRSS2 enzyme are higher during the third trimester. There is a theoretical possibility of an increased risk of late pregnancy complications and vertical transmission due to the COVID-19 infection. Recent case reports indicate extensive fetal vascular malperfusion and parenchymal infarction resulting in a severe loss of a significant percentage of chorionic villi in the placental examination. However, additional investigation and rigorous research are warranted to confirm placental pathology mechanisms concerning COVID-19 to protect maternal and fetal health.

\section{Funding}

The authors received no financial support for the research, authorship, and/or publication of this article.

\section{Conflict of Interest}

The authors indicate no potential conflicts of interest.

\section{Acknowledgment}

We want to thank our host institution for the support.

\section{References}

1 Chowdhury TI, Choudhury TR, Rahman MM, Das TR, Alam J. Spontaneous abortion in pregnancies having COVID-19 infection in Bangladesh: a series of cases. SSRN Electron J January 2021. Doi: 10.2139/ssrn.3760523

2 Rotshenker-Olshinka K, Volodarsky-Perel A, Steiner N, Rubenfeld E, Dahan MH. COVID-19 pandemic effect on early pregnancy: are miscarriage rates altered, in asymptomatic women? Arch Gynecol Obstet 2021;303(03):839-845

3 Cascella M, Rajnik M, Cuomo A. Features, evaluation, and treatment of coronavirus (COVID-19). - PubMed. StatPearls. Published 2021. Accessed April 17, 2021 at: https://pubmed.ncbi.nlm.nih. gov/32150360/

4 Bergsbaken T, Fink SL, Cookson BT. Pyroptosis: host cell death and inflammation. Nat Rev Microbiol 2009;7(02):99-109

5 Cosma S, Carosso AR, Cusato J, et al. Coronavirus disease 2019 and first-trimester spontaneous abortion: a case-control study of 225 pregnant patients. Am J Obstet Gynecol 2021;224(04):391. e1-391.e7

6 Silasi M, Cardenas I, Kwon JY, Racicot K, Aldo P, Mor G. Viral infections during pregnancy. Am J Reprod Immunol 2015;73(03): 199-213

7 Veenstra van Nieuwenhoven AL, Heineman MJ, Faas MM. The immunology of successful pregnancy. Hum Reprod Update 2003; 9(04):347-357

8 Druckmann R, Druckmann MA. Progesterone and the immunology of pregnancy. J Steroid Biochem Mol Biol 2005; 97:389-396
9 Young BC, Stanic AK, Panda B, Rueda BR, Panda A. Longitudinal expression of Toll-like receptors on dendritic cells in uncomplicated pregnancy and postpartum. Am J Obstet Gynecol 2014;210 (05):445.e1-445.e6

10 Piccinni MP, Romagnani S. Regulation of fetal allograft survival by a hormone-controlled Th1- and Th2-type cytokines. Immunol Res 1996;15(02):141-150

11 Di Renzo GC, Giardina I. Coronavirus disease 2019 in pregnancy: consider thromboembolic disorders and thromboprophylaxis. Am J Obstet Gynecol 2020;223(01):135

12 Abou-Ismail MY, Diamond A, Kapoor S, Arafah Y, Nayak L. The hypercoagulable state in COVID-19: incidence, pathophysiology, and management. Thromb Res 2020;194:101-115

13 Whittaker E, Bamford A, Kenny J, et al; PIMS-TS Study Group and EUCLIDS and PERFORM Consortia. Clinical characteristics of 58 children with a pediatric inflammatory multisystem syndrome temporally associated with SARS-CoV-2. JAMA 2020;324(03): 259-269

14 Hosier H, Farhadian SF, Morotti RA, et al. SARS-CoV-2 infection of the placenta. J Clin Invest 2020;130(09):4947-4953

15 Dashraath P, Wong JLJ, Lim MXK, et al. Coronavirus disease 2019 (COVID-19) pandemic and pregnancy. Am J Obstet Gynecol 2020; 222(06):521-531

16 Baud D, Greub G, Favre G, et al. Second-trimester miscarriage in a pregnant woman with SARS-CoV-2 infection. JAMA 2020;323 (21):2198-2200

17 Yan J, Guo J, Fan C, et al. Coronavirus disease 2019 in pregnant women: a report based on 116 cases. Am J Obstet Gynecol 2020; 223(01):111.e1-111.e14

18 Rana MS, Usman M, Alam MM, et al. First trimester miscarriage in a pregnant woman infected with COVID-19 in Pakistan. J Infect 2021;82(01):e27-e28

19 Wong TC, Lee ZY, Sia TLL, Chang AKW, Chua HH. Miscarriage risk in COVID-19 infection. SN Compr Clin Med 2020;2(09):1-4

20 Schjenken JE, Tolosa JM, Paul JW, Clifton VL, Smith R. Mechanisms of maternal immune tolerance during pregnancy. In: Zheng J, ed. Recent Advances Research Human Placenta. Vol. 11. Croatia: InTech; 2012:211-242

21 Buonsenso D, Costa S, Sanguinetti M, et al. Neonatal late onset infection with severe acute respiratory syndrome coronavirus 2 . Am J Perinatol 2020;37(08):869-872

22 Chen L, Li Q Zheng D, et al. Clinical characteristics of pregnant women with Covid-19 in Wuhan, China. N Engl J Med 2020;382 (25):e100

23 Di Mascio D, Sen C, Saccone G, et al. Risk factors associated with adverse fetal outcomes in pregnancies affected by Coronavirus disease 2019 (COVID-19): a secondary analysis of the WAPM study on COVID-19. J Perinat Med 2020;48(09):950-958

24 Mattar CN, Kalimuddin S, Sadarangani SP, et al. Pregnancy outcomes in COVID-19: a prospective cohort study in Singapore. Ann Acad Med Singap 2020;49(11):857-869

25 Hachem R, Markou GA, Veluppillai C, Poncelet C. Late miscarriage as a presenting manifestation of COVID-19. Eur J Obstet Gynecol Reprod Biol 2020;252:614

26 Shmakov RG, Prikhodko A, Polushkina E, et al. Clinical course of novel COVID-19 infection in pregnant women. J Matern Fetal Neonatal Med 2020:1-7

27 Mayeur A, Binois O, Gallot V, et al. First follow-up of art pregnancies in the context of the COVID-19 outbreak. Eur J Obstet Gynecol Reprod Biol 2020;253:71-75

28 Sahin D, Tanacan A, Erol SA, et al. Updated experience of a tertiary pandemic center on 533 pregnant women with COVID-19 infection: a prospective cohort study from Turkey. Int J Gynaecol Obstet 2021;152(03):328-334

29 Michel A-S, De Logiviere V, Schnuriger A, Lefebvre M, Maisonneuve E, Kayem G. Description of a late miscarriage case at 16 Weeks of Gestation associated with a SARS-CoV-2 infection. J Gynecol Obstet Hum Reprod 2021;50(03):102064 
30 D'Antonio F, Sen C, Mascio DDI, et al.On the behalf of the World Association of Perinatal Medicine working group on coronavirus disease 2019. Maternal and perinatal outcomes in high compared to low risk pregnancies complicated by severe acute respiratory syndrome coronavirus 2 infection (phase 2): the World Association of Perinatal Medicine working group on coronavirus disease 2019. Am J Obstet Gynecol MFM 2021;3(04):100329

31 Sentilhes L, De Marcillac F, Jouffrieau C, et al. Coronavirus disease 2019 in pregnancy was associated with maternal morbidity and preterm birth. Am J Obstet Gynecol 2020;223(06):914.e1-914.e15

32 la Cour Freiesleben N, Egerup P, Hviid KVR, et al. SARS-CoV-2 in first trimester pregnancy: a cohort study. Hum Reprod 2021;36 (01):40-47

33 Ayed A, Embaireeg A, Benawadh A, et al. Maternal and perinatal characteristics and outcomes of pregnancies complicated with COVID-19 in Kuwait. BMC Pregnancy Childbirth 2020;20(01):754

34 Shende P, Gaikwad P, Gandhewar M, et al. Persistence of SARSCoV-2 in the first trimester placenta leading to transplacental transmission and fetal demise from an asymptomatic mother. Hum Reprod 2021;36(04):899-906

35 de Vasconcelos Gaspar A, Santos Silva I. SARS-CoV-2 in pregnancy-the first wave. Medicina (Kaunas) 2021;57(03):241

36 Martinez-Perez O, Prats Rodriguez P, Muner Hernandez M, et al; Spanish Obstetric Emergency Group. The association between
SARS-CoV-2 infection and preterm delivery: a prospective study with a multivariable analysis. BMC Pregnancy Childbirth 2021;21 (01):273

37 Pulinx B, Kieffer D, Michiels I, et al. Vertical transmission of SARSCoV-2 infection and preterm birth. Eur J Clin Microbiol Infect Dis 2020;39(12):2441-2445

38 Review WP. Abortion Rates by Country 2021. Accessed April 17, 2021 at: https://worldpopulationreview.com/country-rankings/abortion-rates-by-country

39 Wastnedge EAN, Reynolds RM, van Boeckel SR, et al. Pregnancy and COVID-19. Physiol Rev 2021;101(01):303-318

40 Dhaundiyal A, Kumari P, Jawalekar SS, Chauhan G, Kalra S, Navik U. Is highly expressed ACE 2 in pregnant women "a curse" in times of COVID-19 pandemic? Life Sci 2021;264:118676

41 Poisson TM, Pierone G Jr. Placental pathology and fetal demise at 35 weeks of gestation in a woman with SARS-CoV-2 infection: a case report. Case Rep Womens Health 2021;30:e00289

42 Muyayalo KP, Huang D-H, Zhao S-J, Xie T, Mor G, Liao A-H. COVID19 and Treg/Th17 imbalance: potential relationship to pregnancy outcomes. Am J Reprod Immunol 2020;84(05):e13304

43 Yusuf KK, Dongarwar D, Ibrahimi S, Ikedionwu C, Maiyegun SO, Salihu HM. Expected surge in maternal mortality and severe morbidity among African-Americans in the era of COVID-19 pandemic. Int J MCH AIDS 2020;9(03):386-389 